\title{
Interactive fuzzy satisfying method for optimal multi-objective VAR planning in power systems
}

Y.-L. Chen
C.-C. Liu

Indexing terms: Interactive fuzzy satisfying method, Multi-objective VAR planning problem

\begin{abstract}
The paper presents an interactive fuzzy satisfying method for solving a multi-objective VAR planning problem by assuming that the decision maker (DM) has imprecise or fuzzy goals for each of the objective functions. Through the interaction with the DM, the fuzzy goals of the DM are quantified by eliciting corresponding membership functions. If the DM specifies the reference membership values, the minimax problem is solved for generating a corresponding global noninferior optimal solution for the DM's reference membership values. Then, by considering the current values of the membership functions as well as the objectives, the DM acts on this solution by updating the reference membership values. The interactive procedure continues until the satisfying solution for the DM is obtained. The minimax problem can be easily handled by the simulated annealing (SA) approach which can find a global optimal solution even for the solution space which is nonconvex and the objective functions which are non-differentiable. Results of the application of the proposed method are presented.
\end{abstract}

\section{Introduction}

The purpose of optimal VAR planning lies in providing the system with enough VAR sources so that it is operated in an economically feasible operating condition and the system security margin is enhanced, while load constraints and operational constraints with respect to credible contingencies are met.

In the last decade, numerous methods have been developed for a more systematic approach to VAR planning [1-12]. A common characteristic of these methods is that the value of VAR sources has always been treated as continuously differentiable. Additionally, these methods have expressed the multi-objective VAR planning problem as a single objective optimisation. The most important feature is that the system security margin is not taken into consideration $[1-8,10-12]$.

In this paper optimal VAR planning is formulated as a multi-objective optimisation problem. The objectives consist of three important factors: i.e. the economical operating condition of the system, system security margin, and the voltage deviation of the system. The

(C) IEE, 1994

Paper 1459C (P9), received 25th March 1994

The authors are with the Department of Electrical Engineering, National Taiwan University, Taipei, Taiwan, People's Republic of China

554 operating constraints, load constraints of the system and the new VAR source expansion constraints are also taken into consideration.

In multi-objective optimisation problems (MOP), multiple objectives are usually noncommensurable and cannot be combined into a single objective. Moreover, the objectives usually conflict with each other in that any improvement of one objective can be reached only at the loss of another. Consequently, it is necessary to present a decision maker (DM) for the MOP which implies a trade-off between objectives. The aim of the MOP lies in finding a compromise between satisfying the solution of DM. This means that the DM must select the compromise or satisfying solution from among global noninferior solutions.

In this paper, assuming that the DM has imprecise or fuzzy goals for each of the objective functions in the MOP, an interactive fuzzy satisfying method is presented for solving the multi-objective VAR planning problems.

This paper is summarised as follows:

(i) A proposed method is presented for dealing with the imprecise nature of the DM's judgement in multiobjective VAR planning in power systems.

(ii) Through interaction with the DM, the proposed method is capable of finding a desirable compromise or satisfying solution for a general MOP.

(iii) The simulated annealing (SA) approach is applied for solving the minimax problem and it can search towards a global optimal solution even for nonconvex objective space.

(iv) A formulation of multi-objectives for VAR planning represents a more realistic mathematical model for the actual behaviour of a power system; the proposed method can easily cope with the discrete and nondifferentiable variables in MOP. The salient feature of the proposed method is that it is unnecessary to assume the convexity of the objective functions and the constraint set.

\section{Problem formulation}

A formulation for multi-objective VAR planning is presented in this Section. This formulation treats the problem as a multi-objective mathematical programming problem, which is concerned with the attempt to improve each objective simultaneously. The equality and inequality constraints of the system must, meanwhile, be satisfied.

Here the general multi-objective VAR planning problem is formulated as the following three objective optimisation problems. The objectives consist of three important terms: the economical operating condition of

IEE Proc.-Gener. Transm. Distrib., Vol. 141, No. 6, November 1994 
the system, the system security margin and voltage deviation of system

$$
\begin{aligned}
\min _{u, w \in X} f_{1}(x, u, w) & =K_{e} P_{\text {loss }}(x, u, w) D_{i}+C(w) \\
\max _{u, w \in X} f_{2}(x, u, w) & =S M(x, u, w) \\
& =\frac{\sum_{j \in J_{L}} S_{j}^{\text {limit }}-\sum_{j \in J_{L}} S_{j}^{\text {initial }}}{\sum_{j \in J_{L}} S_{j}^{\text {limit }}} \\
\min _{\boldsymbol{u} . w \in X} f_{3}(x, u, w) & =\max _{i \in J_{L}}\left|V_{i}-V_{i}^{\text {ideal }}\right|
\end{aligned}
$$

subject to

$$
\begin{aligned}
& L(x, u, w)=0 \\
& G(x, u, w) \leqslant 0
\end{aligned}
$$

where

$$
\begin{aligned}
x & =\text { state variable vector } \\
u & =\text { control variable vector } \\
w & =\text { expansion variable vector of the new VAR } \\
& \text { sources } \\
\chi & =\text { a feasible solution region } \\
K_{e} & =\text { converted real power to expense } \\
D & =\text { duration of the system operating time } \\
\left.P_{\text {losss }} \cdot \cdot\right) & =\text { system real power loss } \\
C(w) & =\text { total purchase cost of the new installed VAR } \\
& \text { sources } \\
& =\sum_{i \in \Omega_{L}}\left(d_{i}+s_{c i} q_{c i}+s_{r i} q_{r i}\right) \\
\Omega_{L} & =\text { a set of all candidate buses to install new } \\
& \text { VAR sources } \\
J_{L} & =\text { a set of all load buses } \\
d_{i} & =\text { installed cost at bus } i \\
s_{c i} & =\text { unit costs of capacitor } \\
S_{r i} & =\text { unit costs of reactor } \\
q_{c i} & =\text { added capacitive compensation at bus } i ; \text { it is } \\
& \text { an integer } \\
q_{r i} & =\text { added inductive compensation at bus } i ; \text { it is } \\
S M & \text { an integer } \\
S_{j}^{\text {initial }} & =\text { security margin of the system } \\
S_{j}^{\text {limit }} & =\text { MVA load of bus } j \text { at initial state } \\
V_{i} & =\text { voltage magnitude at bus } i \\
V_{i}^{i d e a} & =\text { ideal specific voltage at bus } i \\
L(\cdot) & =\text { equal constraint set } \\
G(\cdot) & =\text { inequal constraint set }
\end{aligned}
$$

Obadina et al. [13] determined the load limit, $\sum_{j \in J_{L}} S_{j}^{l i m i t}$, of a general multimachine power system.

The equality constraints are the active and reactive power balance load flow equations which hold for every bus of the system. The inequality constraints consist of the available range of the active and reactive generated powers, the bound of the controlled transformer tap change, line flow limits, security bonds on voltage magnitudes, and the limits on voltage angle differences between every pair of buses.

The VAR planning problem consequently becomes a constrained, multi-objective and nondifferentiable optimisation problem (MNOP). In this optimisation problem, it is attempted to minimise the operating cost and voltage deviation, and maximise the system security margin, simultaneously. To maximise the system security margin a system must be able to suffer more load demand

IEE Proc.-Gener. Transm. Distrib., Vol. 141, No. 6, November 1994 without voltage collapse. Put another way, a severe contingency (e.g. line outage etc.) must not cause a voltage collapse.

Fundamental to the MNOP is the Pareto optimal concept, which is also known as a noninferior solution [14]. A noninferior solution of the MNOP is that any improvement of one objective can be reached only at the loss of another. Mathematically, formal definitions of local and global noninferior solutions to the MNOP are given in the following.

Definition (local noninferior solution): $z^{*}$ is said to be a local noninferior solution of MO if there exists an $\varepsilon>0$ such that in the neighbourhood $N\left(z^{*}, \varepsilon\right)$ of $z^{*}$ there exists no other feasible $z$ (i.e. $z \in N\left(z^{*}, \varepsilon\right)$ ) such that $f(z) \leqslant f\left(z^{*}\right)$, meaning $f_{i}(z) \leqslant f_{i}\left(z^{*}\right)$ for all $i=1,2, \ldots, m$ with strict inequality for at least one $i$. The $f\left(z^{*}\right)$ is said to be a local noninferior solution of $\mathrm{MO}$ in objective space.

Definition (global noninferior solution): $z^{*}$ is said to be a global noninferior solution of MO if there exists no other feasible $z$ (i.e., $z \in \chi$ ) such that $f(z) \leqslant f\left(z^{*}\right)$, meaning $f_{i}(z) \leqslant f_{i}\left(z^{*}\right)$ for all $i=1,2, \ldots, m$ with strict inequality for at least one $i$. The $f\left(z^{*}\right)$ is said to be a global noninferior solution of MO in objective space.

Usually, the global noninferior solutions consist of an infinite number of points, and the DM must be presented for some kind of subjective judgement. The DM must select a compromise or satisfying solution from among the global noninferior solutions.

In this paper, assuming that the DM has imprecise or fuzzy goals for each of the objective functions in the MNOP, we present an interactive fuzzy satisfying method based on SA for solving a general multi-objective VAR planning problem. It is described in the following Section.

\section{$3 \quad$ Interactive fuzzy satisfying decision making}

Assuming that the DM has imprecise or fuzzy goals for each of the objectives in the MNOP, a fuzzy goal expressed by the DM can be quantified by drawing out a corresponding membership function. To elicit a membership function $\mu_{f_{i}}(z)$ from the DM for each objective function $f_{i}(z), i=1,2, \ldots, m$, we first estimate (or calculate) the individual minimum $f_{i}^{\min }$ and maximum $f_{i}^{\max }$ of each objective function $f_{i}(z)$ under given constraints by the experiences of the DM. By taking account of the estimated $f_{i}^{\min }$ and $f_{i}^{\max }$, the DM must select the subjective membership function $\mu_{f}(z)$, which is a strictly monotonically decreasing function with respect to $f_{i}(z)$. Here, in a minimisation problem, it is assumed that $\mu_{f}(z)=0$ or $\rightarrow 0$, if $f_{i}(z) \geqslant f_{i}^{\max }$ and $\mu_{f}(z)=1$ or $\rightarrow 1$, if $f_{i}(z) \leqslant f_{i}^{\min }$, where $f_{i}^{\max }$ and $f_{i}^{\min }$ are an unacceptable level and the desirable level for $f_{i}(z)$, respectively. In contrast with the minimisation problem, in a maximisation problem, it is assumed that $\mu_{f_{i}}(z)=0$ or $\rightarrow 0$, if $f_{i}(z) \leqslant f_{i}^{\text {min }}$ and $\mu_{f_{i}}(z)=1$ or $\rightarrow 1$, if $f_{i}(z) \geqslant f_{i}^{\max }$.

Having determining the membership functions for each of the objective functions, in order to generate a satisfying solution, the DM is asked to specify the reference (desirable) levels of achievement of the membership functions, called reference membership values [15]. For the DM's reference membership values $\bar{\mu}_{f_{i}}, i=1,2, \ldots$, $m$, the corresponding global optimal solution, which is in a sense close to the DM's requirement, can be obtained 
by solving the following minimax problem $[16-18]$ :

$$
\min _{z \in \chi}\left\{\max _{i=1,2, \ldots, m}\left(\vec{\mu}_{f i}-\mu_{f_{i}}(z)\right)\right\}
$$

It should be emphasised here that it is possible for DM to improve the solution by updating the reference membership values $\bar{\mu}_{f_{i}}$ when the DM is not satisfied with the current global non-inferior solution. In the following theorem, we prove a global optimal solution of the minimax problem (expr. 6) and also a global optimal solution of the MNOP.

Theorem 1: If $\bar{z}$ is not a global noninferior solution to the MNOP then $\bar{z}$ is not a global optimal solution to the minimax problem (expr. 6) for some $\bar{\mu}_{f}=\left(\bar{\mu}_{f_{1}}, \bar{\mu}_{f_{2}}, \ldots\right.$, $\left.\bar{\mu}_{f_{m}}\right)$.

Proof: Assume that $\bar{z}$ is not a global noninferior solution to the MNOP, then there exists some $z \in \chi$ such that

$$
\begin{aligned}
f(z) & <f(\bar{z}) \\
\mu_{f}(z) & >\mu_{f}(\bar{z}) \\
\bar{\mu}_{f}-\mu_{f}(z) & <\bar{\mu}_{f}-\mu_{f}(\bar{z}) \\
\max _{i}\left[\bar{\mu}_{f i}-\mu_{f i}(z)\right] & <\max _{i}\left[\bar{\mu}_{f_{i}}-\mu_{f i}(\bar{z})\right]
\end{aligned}
$$

Then it holds that

$$
\min _{z \in x} \max _{i}\left[\bar{\mu}_{f_{i}}-\mu_{f_{i}}(z)\right]<\max _{i}\left[\bar{\mu}_{f_{i}}-\mu_{f_{i}}(\bar{z})\right]
$$

Thus, the theorem is proved.

Theorem 2: If $z^{*}$ is a global noninferior solution to the MNOP then $z^{*}$ is a global optimal solution to the minimax problem (expr. 6) for some $\bar{\mu}_{f}=\left(\bar{\mu}_{f_{1}}, \bar{\mu}_{f_{2}}, \ldots\right.$, $\left.\bar{\mu}_{f_{m}}\right)$.

Proof: Assume that $z^{*}$ is a global noninferior solution to the MNOP, then there exists all $z \in \chi$ such that

$$
\begin{aligned}
f\left(z^{*}\right) & \geqslant f(z) \\
\mu_{f}\left(z^{*}\right) & \leqslant \mu_{f}(z) \\
\bar{\mu}_{f}-\mu_{f}\left(z^{*}\right) & \geqslant \bar{\mu}_{f}-\mu_{f}(z) \\
\max _{i}\left[\bar{\mu}_{f_{i}}-\mu_{f_{i}}\left(z^{*}\right)\right] & \geqslant \max _{i}\left[\bar{\mu}_{f_{i}}-\mu_{f_{i}}(z)\right]
\end{aligned}
$$

Thus, the theorem is proved.

Theorems 1 and 2 guarantee the fact that $z^{*}$ is a global optimal solution to the minimax problem (expr. 6) and also a global noninferior solution to the MNOP.

In the following, we construct the interactive fuzzy satisfying method to derive the satisfying solution for the DM from the noninferior solution set in the MNOP. The resulting solution would be very much a compromise solution for the DM. The steps of the proposed interactive algorithm can be stated as follows and the steps marked with an asterisk involve interaction with the DM.

Step 0: Input the problem data and set the interactive index $v=0$.

Step $1^{*}$ : Estimate (or calculate) the $f_{i}^{\min }$ and $f_{i}^{\max }$, for $i=1,2, \ldots, m$ by the experiences of the DM.
Step 2*: Draw out a membership function $\mu_{f i}(z)$ from the DM for each of the objective functions.

Five types of membership functions are selected (see Fig. 1):

a linear membership function

$b$ convex exponential membership function

$c$ concave exponential membership function

$d$ hyperbolic membership function

$e$ piecewise-linear membership function.
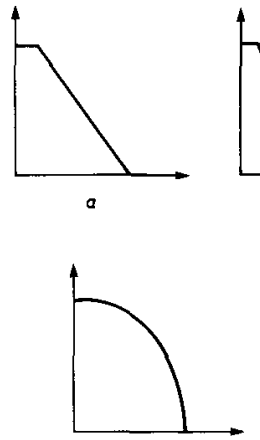

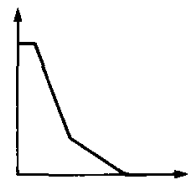

$b$

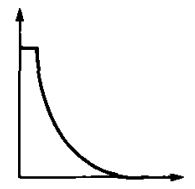

$c$
Fig. 1 Five types of membership functions

a Linear membership function

$b$ Piecewise-linear membership function

c Convex exponential membership function

$d$ Concave exponential membership function

e Hyperbolic membership function

Step 3: Set the initial reference membership values $\bar{\mu}_{f_{i}}^{(v)}=1, i=1,2, \ldots, m$.

Step 4: Solve the minimax problem using the simulated annealing technique to obtain the corresponding global optimal solution with respect to $\bar{\mu}_{f_{i}}^{(v)}$

$$
\min _{z \in \chi}\left\{\max _{i=1.2, \ldots, m}\left(\bar{\mu}_{f_{i}}^{(v)}-\mu_{f_{i}}(z)\right)\right\}
$$

Step 5*: Observe $\mu_{f_{i}}^{(v)}\left(z^{*}\right)$ and $f_{i}^{(v)}\left(z^{*}\right), i=1,2, \ldots, m$.

If $\mu_{f_{i}}^{(v)}\left(z^{*}\right)$ and $f_{i}^{(v)}\left(z^{*}\right), i=1,2, \ldots, m$ are satisfactory, go to next step; otherwise, set $v=v+1$ and select new reference membership values, $\bar{\mu}_{f_{i}}^{(v)}, i=1,2, \ldots, m$. Go to Step 4.

Step 6: Output the best compromise solution, $z^{(v)}$, $f^{(v)}\left(z^{*}\right)$ and $\mu_{f_{i}}\left(z^{*}\right)$ for the DM.

In Step 1, the DM must estimate the $f_{i}^{\min }$ and $f_{i}^{\max }$ for each of the objective functions by the DM's experiences. Then a membership function $\mu_{f_{i}}(z)$ must be elicited by the DM for each of the objective functions. Usually, four types of membership functions can be chosen. A global noninferior solution which is close to the DM's reference membership values will be obtained in Step 4. In Step 5, the DM acts on this solution by either updating the reference membership values or not. In Step 4 and Step 5, continue until the best compromise, the global noninferior solution is found.

It may be worthwhile to note here that the proposed interactive algorithm offers several advantages.

(a) The DM does not give an accurate goal for each of the objectives in MNOP.

(b) The DM can change the reference membership values at each iteration, thus achieving a certain degree of flexibility in solving the decision-making problem.

IEE Proc.-Gener. Transm. Distrib., Vol. 141, No. 6, November 1994 
(c) By communicating with the DM, the solution produced by the algorithm should improve and possibly converge to the most satisfactory solution.

\section{$4 \quad$ Simulated annealing}

In this Section, we briefly describe the algorithm of the simulated annealing approach as follows (see Reference 21 for more details). A parameter $T$, temperature, is defined in this algorithm.

\subsection{Algorithm: Simulated annealing}

Step 1: Randomly choose an initial condition (solution).

Step 2: Generate a feasible point neighbour of the current point from the solution space.

Step 3: Evaluate the increase in the cost $\Delta C$.

Step 4: If $\Delta C \leqslant 0$ then accept the new solution point and go to Step 6.

Step 5: A random number $r$, uniformly distributed in the interval $[0,1)$, is chosen. If $\exp (-\Delta C / T)>r$ then accept the new point; otherwise, the new point is discarded.

Step 6: If the moves are not finished, go to Step 2.

Step 7: Cooling down temperature, $T=\beta \times T$.

Step 8: If $T>T_{\min }$, go to Step 2.

Step 9: Output global optimal solution.

The advantages of the SA approach being applied to optimal VAR source planning lie in that it is both capable of handling a mixed-integer nonlinear programming problem and also can search toward a (near) global optimal solution [20-24].

\section{Decision strategy in VAR planning problem}

In this Section, we present some decision strategy for the decision maker to design the satisfactory membership functions for each objectives and to set or change the reference membership values in the VAR planning problem. At the same time, we propose a weak busoriented and a heavy load-oriented criterion to choose the buses for installing new VAR sources.

\subsection{Design membership function}

The power of any fuzzy-set-based method depends very much on the design of the membership functions. Hence, it is crucial to the design of the membership functions for each objective. Usually, a membership function for each of the objective functions is drawn out by the experiences and intuitive knowledge of the DM or planner. In the VAR planning problem, three important objectives are taken into consideration. To design a good membership function, the following suggestions are considered.

5.1.1 Cost function: This objective is to minimise the total cost of the system. A linear membership function (see Fig. 1a) is a good choice for this objective because it has no strict limit. In the linear membership function, an unacceptable level, $f_{i}^{\max }$, set to be the initial operating cost of the system, and a desirable level, $f_{i}^{\text {min }}$, set to be the reduced percentage (e.g. 35\%) of $f_{i}^{\max }$ are the good estimations.

5.1.2 System security margin: Maximising the system security margin means that a system can suffer more load demand without voltage collapse. Another meaning is that a severe contingency (e.g. line outage) will not cause a voltage collapse. A convex exponential membership function (see Fig. 1c) is a good choice for a system security margin objective because this objective will damage the system security. For the convex exponential membership function in the maximisation problem, the membership value is increased slowly as the objective of the system security margin increases to near an unacceptable level, and the membership value is increased quickly as the objective increases to near a desirable level. This result allows the system a certain safety security margin in after-VAR planning. For the above reason, the hyperbolic membership function will also be a good choice for this objective.

According to the definition of the SM, a stable operating condition of the system, the SM must take on values between 0 and 1 . It is obvious that, in order to set an unacceptable level, $f_{i}^{\min }=0$ is suitable, and to set a desirable level, $f_{i}^{\max }$, is suitable, estimated by the DM or planner (e.g. $f_{i}^{\max }=0.4$ ).

5.1.3 Voltage magnitude deviation: To minimise this objective means trying to push the values of the voltage toward a hard limit. In general, an ideal range of load bus voltage is between 0.95 and 1.05 p.u. For this reason, a linear or a piecewise-linear membership function will be a good selection. In VAR planning problems, the authors suggest that the choice of a piecewise-linear membership function (see Fig. 1b) is more suitable than a linear function. An unacceptable level, $f_{i}^{\max }$, set close to 0.05 p.u., and a desirable level, $f_{i}^{\text {min }}$, set close to zero, are the best choices. In the piecewise-linear membership function for the voltage deviation objective, the absolute value of the slope of the segments of the piecewise-linear curve near the unacceptable level is designed to be smaller, and the absolute value of the slope of the segments near the desirable level is chosen to be larger.

\subsection{Criterion of set reference membership value}

The interactive fuzzy satisfying method can solve a satisfactory or compromise global noninferior solution of the DM who sets or changes the reference membership values, $\bar{\mu}_{f_{i}}$. In the interactive procedure, the initial reference values are all set to 1 , then by solving the minimax problem (expr. 6) the corresponding global optimal solution can be obtained. Then by considering the current values of the membership functions as well as the objectives, the DM sets a larger value of $\bar{\mu}_{f_{i}}$ in order to improve the $i$ th objective, $f_{i}(z)$, and sets smaller values to the other reference membership values. The criterion is the improvement of $i$ th objective with the set of larger reference membership values of $\bar{\mu}_{f_{i}}$.

\subsection{Criterion of selection candidate buses}

In the past, the determination of the candidate buses for installing new VAR sources was based on the experiences of the planner or the environmental limit. In this paper, we present two criteria, a weak bus-oriented and a heavy load bus-oriented, in order to determine the candidate buses. 
5.3.1 Weak bus-oriented criterion: A system may be voltage unstable if it includes at least one voltage unstable bus [25], and appropriate VAR planning can enhance the system security margin [9]. For the above reason, the weak bus is an appropriate bus for installing new VAR sources in the system. Using this criterion, we select the weak buses as the candidate buses.

5.3.2 Heavy load bus-oriented criterion: This criterion is based on the concept of intuition. A heavy load bus is usually a very voltage-sensitive bus and installing new VAR sources is necessary. The heavy load buses are then the primary choices as candidate buses.

\section{Numerical examples}

Consider the application of the proposed method to the AEP 14-bus system [11]. The heavy load conditions of the system are shown in Tables 1 and 2. The constant

Table 1: Estimate of heavy load operating condition (AEP 14 bus)

\begin{tabular}{rllrrr}
\hline Bus & $\begin{array}{l}\text { Bus } \\
\text { mag. }\end{array}$ & $\begin{array}{l}\text { Voltage } \\
\text { ang. }\end{array}$ & Bus & Power* & $\begin{array}{l}\text { Static } \\
\text { shunt }\end{array}$ \\
\hline & & & MW & MVAR & \\
1 & 1.06 & 0.0 & \multicolumn{1}{l}{-1} & \multicolumn{1}{c}{-} & \\
2 & 1.045 & 0.0 & 40.0 & 0.0 & \\
3 & 1.01 & 0.0 & -94.2 & -19.0 & \\
4 & 1.0 & 0.0 & -57.8 & -23.9 & \\
5 & 1.0 & 0.0 & -47.6 & -1.6 & \\
6 & 1.01 & 0.0 & 0.0 & 0.0 & \\
7 & 1.0 & 0.0 & 0.0 & 0.0 & \\
8 & 1.01 & 0.0 & 0.0 & 0.0 & \\
9 & 1.0 & 0.0 & -29.5 & -16.6 & 0.19 \\
10 & 1.0 & 0.0 & -29.5 & -5.8 & \\
11 & 1.0 & 0.0 & -13.5 & -5.8 & \\
12 & 1.0 & 0.0 & -36.1 & -11.6 & \\
13 & 1.0 & 0.0 & -23.5 & -15.8 & \\
14 & 1.0 & 0.0 & -14.9 & -10.0 & \\
\hline
\end{tabular}

* for load

$\dagger 100$ MVAR base

Table 2: Regulated bus data

\begin{tabular}{lllrr}
\hline Bus & $\begin{array}{l}\text { MVAR } \\
\text { min. }\end{array}$ & $\begin{array}{l}\text { Limits } \\
\text { max. }\end{array}$ & $\begin{array}{l}\text { MW } \\
\text { min. }\end{array}$ & $\begin{array}{l}\text { Limits } \\
\text { max. }\end{array}$ \\
\hline 2 & -6.0 & 24.0 & 30.0 & 70.0 \\
3 & -6.0 & 24.0 & 0.0 & 0.0 \\
6 & -6.0 & 24.0 & 0.0 & 0.0 \\
8 & -6.0 & 24.0 & 0.0 & 0.0 \\
\hline
\end{tabular}

power model is applied to all load demands. To determine the maximum MVA load demand, $\sum_{j \in J_{L}} S_{j}^{\text {limit }}$, the components of the distribution vector $\rho$ are chosen to be $\rho_{i}=S_{i}^{\text {initial }} / \sum_{j \in J_{L}} S_{j}^{\text {initial }}$, and the participation factors of the generating unit are chosen as in Reference 11.

In order to determine the candidate buses for installing new VAR sources, the identifying weak bus method, proposed by Obadina $e t$ al. [26] is applied to identify the system weak buses. In the study, buses $9,11,12,13$ and 14 are the weak buses, bus 4 is a heavy load bus; and they are all selected as candidate buses for installing new VAR sources (i.e. $\Omega_{L}=\{4,9,11,12,13,14\}$ ). One bank of the VAR source is set at 3 MVAR, the specific voltage $V_{i}^{\text {ideal }}$ of all load buses is set at 1 p.u., and the following parameters are used: power loss cost weight $\left(K_{e}=N T\right.$ $\$ 2.31 / \mathrm{kW} \mathrm{h}, D_{i}=10(y) \times 365 \times 0.5 \times 8(h)$ ), VAR source cost weight $\left(s_{c i}=N T \$ 11385 / \mathrm{bank}, s_{r i}=N T \$ 16400 /\right.$ bank and $d_{i}=N T \$ 420000 /$ location), and $q_{\mathrm{c} i}^{\max }$ and $q_{r i}^{\max }$ are all limited to 90 MVAR ( 30 banks). The $K_{e}$ param- eter is the average cost of real power in Taiwan at this time. The lifetime of the VAR compensator is determined as ten years. The duration of the summer peak load is about 6 months/year and 8 hours/day in Taiwan, and the other cost parameters are all estimated in real life in Taiwan.

In the following, some of the interaction processes for solving the multi-objective VAR planning problems under SUN SPARC station 2 in the computer centre of National Taiwan University are explained with the aid of some of the computer outputs.

Some of the membership functions for each of the objectives which are derived by interaction with the DM are shown below.

An interactive fuzzy satisfying method for solving the multi-objective VAR planning problems

Input the name of planning system ? = AEP $-14 \mathrm{bus}$

Initial state of the system

(1) $f 1=1241.10$ system cost (million NT\$)

(2) $f 2=0.09461$ system security margin

(3) $f 3=0.19252$ voltage deviation (p.u.

Draw out a corresponding membership function for each of the objective functions

$L=$ linear membership function

$V=$ convex exponential membership function

$C=$ concave exponential membership function

$H=$ hyperbolic membership function

$P=$ piecewise-linear membership function

$M(f)=$ membership function

$f_{-} 0=$ unacceptable level

$f_{-} 1=$ desirable level

(1) Input the membership function type of $f 1, M 1(f)=$ ? $L$

Input two points $\left(f_{-} 0, f_{-} 1\right)$ such that

$M 1\left(f_{0} 0\right)=0.0, f_{0}=$ ? 1241.1

$M 1(f 1)=10, f=? \quad 12411 * 0.65$

(2) Input the membership function type of $f 2, \mathrm{M} 2(f)=$ ? $V$

Input three points $\left(f_{-} 0, f_{-} 0.5, f_{-} 1\right)$ such that

$\mathrm{M} 2\left(f_{-} 0\right)=0.0 \quad, f_{-} 0=? \quad 0.0$

$M 2(f 0.5)=0.5, f 0.5=? \quad 0.25$

$M 2(f-1)=1.0, f 1$

(3) Input the membership function type of $f 3, M 3(f)=$ ? $P$

Input four points such that

$M 3\left(f_{-} 0\right)=0.0 \quad, f_{-} 0=? \quad 0.06$

$M 3\left(f_{-}\right)=1.0 \quad, f_{-} 1=? \quad 0.01$

$M 3\left(f_{-} a\right)=? \quad 0.5, f_{-} a=? \quad 0.04$

$M 3\left(f_{-} b\right)=? \quad 0.1, f_{-} \mathrm{b}=? \quad 0.05$

Would you want to change the membership function type? $=\mathrm{NO}$

The objective of the system cost is elicited as a linear membership function. An unacceptable level, $f_{1}^{\max }$, is set as initial cost, and a desirable level, $f_{1}^{\text {min }}$, is determined as $f_{1}^{\max } \times 0.65$. This means that the DM's goal is a reduction of $35 \%$. An exponential membership function is expressed for the objective of the system security margin. An unacceptable level, $f_{2}^{\mathrm{min}}$, is set at zero, and a desirable level, $f_{2}^{\max }$, is chosen as 0.4 which is the system security margin of $40 \%$. The membership function value 0.5 is set as the system with $25 \%$ security margin. This design is strictly for system security in heavy loading conditions. The voltage deviation objective is drawn out as a piecewise-linear membership function.

An interactive fuzzy satisfying decision-making process for this example is illustrated below.

IEE Proc.-Gener. Transm. Distrib., Vol. 141, No. 6, November 1994 
WAITING .........

\begin{tabular}{|c|c|c|c|}
\hline RMV & membership & | objective & \\
\hline $\begin{array}{l}R M 1=1.0 \\
R M 2=1.0 \\
R M 3=1.0\end{array}$ & $\begin{array}{l}M 1(f)=0.60613 \\
M 2(f)=0.69177 \\
M 3(f)=0.64577\end{array}$ & $\begin{array}{l}f 1=977.791 \\
f 2=0.31819 \\
f 3=0.03125\end{array}$ & $\begin{array}{l}\text { (million NT\$) } \\
\text { (security margin) } \\
\text { (p.u.) }\end{array}$ \\
\hline
\end{tabular}

$* * *($ CPU time $=943 \mathrm{~s}) * * *$

Are you satisfied with the current noninferior solution? $=\mathrm{NO}$

Input your reference membership values $(R M V)$ for each of the membership function:

$\begin{array}{lllll}R M 1=? \quad 0.660 & R M 2=? & 0.710 & R M 3 & =? \quad 0.700\end{array}$

WAITING .........

Global noninferior solution (interaction 1)

\begin{tabular}{c|cll}
\hline RMV & membership & objective & \\
\hline$R M 1=0.660$ & $M 1(f)=0.60194$ & $f 1=979.610$ & (million NT\$) \\
$R M 2=0.710$ & $M 2(f)=0.67570$ & $f 2=0.31335$ & (security margin) \\
$R M 3=0.700$ & $M 3(f)=0.67385$ & $f 3=0.02957$ & (p.u.) \\
\hline
\end{tabular}

$* * *(\mathrm{CPU}$ time $=324 \mathrm{~s}) * * *$

Are you satisfied with the current noninferior solution? $=$ NO

Input your reference membership values $(R M V)$ for each of the membership function

$R M 1=? \quad 0.660 \quad R M 2=? \quad 0.700 \quad R M 3=? \quad 0.710$

WAITING .........

Global noninferior solution (interaction 2)

\section{$R M V$ | membership | objective}

$R M 1=0.660 \quad M 1(f)=0.60393 \quad f 1=978.745 \quad$ (million NT\$)

$R M 2=0.700 \quad M 2(f)=0.67676 \quad f 2=0.31367 \quad$ (security margin)

$\begin{array}{llll}R M 2=0.700 & M 2(f)=0.67676 & f 2=0.31367 & \text { (security } \\ R M 3=0.710 & M 3(f)=0.67678 & f 3=0.02939 & \text { (p.u.) }\end{array}$

***(CPU time $=298 \mathrm{~s}) * * *$

Are you satisfied with the current noninferior solution? $=$ YES

The following values are your satisfying solution:

\begin{tabular}{cll}
\hline membership & objective function \\
\hline$M 1(f)=0.60393$ & $f 1=978.745$ & (million NT\$) \\
$M 2(f)=0.67676$ & $f 2=0.31367$ & (security margin) \\
$M 3(f)=0.67678$ & $f 3=0.02939$ & (p.u.)
\end{tabular}

The loss reduce rate $=21.38 \mathrm{c} / \mathrm{c}$

The cost reduce rate $=21.14 \mathrm{c} / \mathrm{c}$

For the initial reference membership values, $\bar{\mu}_{f_{i}}=1$, the minimax problem is solved to obtain a global noninferior solution. The DM then considers the current values of the membership functions as well as the objective functions in order to choose to update the reference membership values or not. If the DM is not satisfied with the current solution, the DM updates the reference membership values to improve some objectives at the expense of other objectives. In this study, the DM give a larger value to the reference membership value $\bar{\mu}_{f_{3}}=0.71$ in order to improve the voltage performance at the expense of the cost objective, which is given a smaller value of $\bar{\mu}_{f_{1}}=$ 0.66 , causing little expense to the system security margin objective. The same procedure continues according to this scheme until the DM is satisfied with the current optimal solution. At the second interaction, the best compromise and satisfying solution of the DM is obtained in this example. The results of installing new VAR sources for each interaction are given in Table 3. This study required $1565 \mathrm{~s}$ total computer time on a SUN SPARC station 2 computer to obtain the optimal solution. Although the solution algorithm wastes time in obtaining the optimal solution, it is unimportant when applied to

Table 3: Results of the installing new VAR sources (banks)

\begin{tabular}{|c|c|c|c|c|c|c|}
\hline is no. & 4 & 9 & 11 & 12 & 13 & 314 \\
\hline itial & 26 & 4 & 5 & 4 & & \\
\hline Interaction $1\left(\bar{\mu}_{11}^{(1)}=0.66, \bar{\mu}_{z}^{(1)}=0.71, \bar{\mu}_{z}^{(1)}\right.$ & 26 & 3 & 6 & 4 & & \\
\hline Interaction $2\left(\tilde{\mu}_{t_{1}}^{(1)}=0.66, \tilde{\mu}_{t_{2}}^{(2)}=0.70, \mu_{t_{3}}^{(2)}\right.$ & 26 & 2 & 6 & 3 & & \\
\hline
\end{tabular}

Interaction $2\left(\bar{\mu}_{t_{1}}^{(2)}=0.66, \bar{\mu}_{i 2}^{(2)}=0.70, \mu_{t_{3}}^{(2)}=0.71\right) \quad 26 \quad 2 \quad 6 \quad 3 \quad 7$

One bank = 3 MVAR

planning problems, because obtaining the optimal solution is more attractive than minimising the computation time involved in planning problems. Meanwhile, the authors believe that a faster computer will be obtainable in the future and computation time will thus not be a problem.

\section{Conclusion}

In this paper, an interactive fuzzy satisfying method was proposed, to deal with the imprecise or fuzzy goals of the DM in multi-objective VAR planning problems, considering three objectives: an economical operating condition, the system security margin, and voltage deviation. These are all mostly concerned with optimal VAR planning problems. The salient features of the proposed method are: $(a)$ it allows the DMs to learn from the available information or simply 'change their mind', $(b)$ it guarantees that a global noninferior solution, which corresponds to the reference membership values derived by the DM, will be generated at each iteration, and $(c)$ by updating the DM's reference membership values, the proposed method demonstrates that the best compromise solution will be obtained by the interactive scheme.

In fact, the proposed method will, hopefully, become an efficient tool for man-machine interactive fuzzy decision making under multiple conflict objectives.

\section{References}

1 HAPP, H.H., and WIRGAU, K.A.: 'Static and dynamic VAR compensation in system planning', IEEE Trans., 1978, PAS-97

2 FERNANDES, R.A., LANGE, F., BURCHETT, R.C., HAPP, H.H FERNANDES, R.A., LANGE, F., BURCHETT, R.C., HAPP, H.H.,
and WIRGAU, K.A.: 'Large scale reactive power planning', IEEE Trans., 1983, PAS-102, pp. 1083-1088

3 KISHORE, A., and HILL, E.F.: 'Static optimization of reactive power sources by use of sensitivity parameters', IEEE Trans., 1971, PAS-90, pp. 1166-1173

4 IBA, K., SUZUKI, H., SUZUKI, K.I., and SUZUKI, K.: 'Practical reactive power allocation/operation planning using successive linear programming', IEEE Trans., 1988, PWRS-3, (2), pp. 558-566

5 SACHDEVA, S.S., and BILLINTON, R.: 'Optimum network VAR planning by nonlinear programming', IEEE Trans., 1973, PAS-92, pp. $1217-1225$

6 AOKI, K., FAN, M., and NISHIKORI, A.: 'Optimal VAR planning by approximation method for recursive mixed-integer linear proby approximation method for recursive mixed-integer linear

gramming', IEEE Trans., 1988, PWRS-3, (4), pp. 1741-1747
7 LEBOW, W.M., ROUHANI, R., NADIRA, R., USORO, P.B., MEHRA, R.K., SOBIESKI, D.W., PAL, M.K., and BHAVARAJU, M.P.: 'A hierarchical approach to VAR optimization in system planning', IEEE Trans., 1985, PAS-104, (8), pp. 2015-2057

8 OPOKU, G.: 'Optimal power system VAR planning', IEEE Trans., 1990, PWRS-5, (1), pp. 53-59

9 OBADINA, O.O., and BERG, G.J.: 'VAR planning for power system security', IEEE Trans., 1989, PWRS-4, (2), pp. 677-686

10 HONG, Y.Y., SUN, D.I., LIN, S.Y., and LIN, C.J.: 'Multi-year multi-case optimal VAR planning', IEEE Trans., 1990, PWRS-5, (4), pp. 1292-1301

11 ABDUL-RAHMAN, K.H., and SHAHIDEHPOUR, S.M., 'A fuzzybased optimal rective power control'. Presented at IEEE/PES 1992 Summer Meeting, paper 92 SM 402-8 PWRS, Seattle, WA, USA. 1992 
12 ABDUL-RAHMAN, K.H., and SHAHIDEHPOUR, S.M.: 'Application of fuzzy sets to optimal reactive power planning with security constraints'. IEEE 1993 PICA paper

13 OBADINA, O.O., and BERG, G.J.: 'Determination of voltage stability limit in multimachine power systems', IEEE Trans., 1988, PWRS-3, (4), pp. 1545-1554

14 CHU, K.-C.: 'On the noninferior set for the systems with vectorvalued objective function', IEEE Trans., 1970, AC-15, pp. 591-593

15 WIERZBICKI, A.P.: "The use of reference objectives in multiobjective optimization-theoretical implications and practical experiences'. Int. Inst. Applied System Analysis, Laxenburg, Austria, Working Paper WP-79-66, 1979

16 SAKAWA, M., and YANO, H.: 'An interactive fuzzy satisfying method using augmented minimax problems and its application to environmental systems', IEEE Trans., 1985, SMC-15, (6), pp. 720729

17 SAKAWA, M., YANO, H., and YUMINE, T.: 'An interactive fuzzy satisfying method for multiobjective linear-programming problems and its application', IEEE Trans., 1987, SMC-17, (4), pp. 654-66

18 CHANKONG, V., and HAIMES, Y.Y.: 'Multiobjective decision making: theory and methodology' (North-Holland, New York, 1983)
19 LASDON, L.S.: 'Optimization theory for large systems' (MacMillan, London, 1970)

20 AARTS, E., and KORST, J.: 'Simulated annealing and Boltzmann machines' (John Wiley, 1989)

21 IRVING, M.R., and STERLING, M.J.H.: 'Optimal network tearing using simulated annealing', IEE Proc. $C, 1990,137,(1)$, pp. 69-72

22 CHIANG, H.D., WANG, J.C., COCKINGS, O.R., and SHIN, H.D.: 'Optimal capacitor placements in distribution systems: Part 1: A new formulation and the overall problem', IEEE Trans., 1990, PWRD-5, (2), pp. 634-642

23 CHIANG, H.D., WANG, J.C., COCKINGS, O.R., and SHIN, H.D.: 'Optimal capacitor placements in distribution systems: Part 2: Solution algorithms and numerical results', IEEE Trans., 1990 , PWRD-5, (2), pp. 643-649

24 HSIAO, Y.T., LIU, C.C., CHIANG, H.D., and CHEN, Y.L.: 'A new approach for optimal VAR sources planning in large electric power systems', IEEE Trans., 1993, PWRS-8, (3), Pp. 988-996

25 CALVAER, A.: 'Voltage stability and voltage collapse', CIGRE Report, Paper 38.02, 1985

26 OBADINA, O.O., and BERG, G.J.: 'Identifying electrically weak and strong segments of a power system from a voltage stability viewpoint', IEE Proc. C, 1990, 137, (3), pp. 205-212 